\title{
On the Space of KdV Fields
}

\author{
ATSUSHI NAKAYASHIKI \\ Department of Mathematics, Kyushu University, Ropponmatsu 4-2-1, Fukuoka 810-8560, \\ Japan.e-mail:6vertex@math.kyushu-u.ac.jp
}

Received: 3 April 2009 / Revised: 11 May 2009 / Accepted: 27 May 2009

Published online: 16 June 2009 - (C) The Author(s) 2009. This article is published with open access at Springerlink.com

\begin{abstract}
The space of functions $A$ over the phase space of $\mathrm{KdV}$-hierarchy is studied as a module over the ring $\mathcal{D}$ generated by commuting derivations. A $\mathcal{D}$-free resolution of $A$ is constructed by Babelon, Bernard and Smirnov by taking the classical limit of the construction in quantum integrable models assuming a certain conjecture. We propose another $\mathcal{D}$-free resolution of $A$ by extending the construction in the classical finite dimensional integrable system associated with a certain family of hyperelliptic curves to infinite dimension assuming a similar conjecture. The relation between the two constructions is given.
\end{abstract}

Mathematics Subject Classification (2000). 37K20, 17B69, $17 \mathrm{~B} 37$.

Keywords. KdV hierarchy, boson-fermion correspondence, space of fields, D-module.

\section{Introduction}

In [1] Babelon, Bernard and Smirnov (BBS), by considering the classical limit of a model in two dimensional integrable quantum field theory, have studied the space of $\mathrm{KdV}$ fields $A=\mathbb{C}\left[u, u^{\prime}, \ldots\right]$ as a module over the ring of commuting derivations $\mathcal{D}=\mathbb{C}\left[\partial_{1}, \partial_{3}, \ldots\right]$, where $\partial_{i}$ acts on $u$ according as the KdV-hierarchy (see Section 2):

$$
\partial_{i} u=S_{i+1}^{\prime}(u), \quad S_{i+1}(u) \in A .
$$

Assuming the conjecture that $A$ is generated over $\mathcal{D}$ by $\mathbb{C}\left[S_{2}, S_{4}, \ldots\right]$ they have constructed a $\mathcal{D}$-free resolution of $A$. In particular all $\mathcal{D}$-linear relations among monomials of $\left\{S_{2 i}\right\}$ are determined. They are called null vectors in [1]. For example the first two non-trivial null vectors are

$$
\partial_{3} S_{2}-\partial_{1} S_{4}=0, \quad \partial_{1}^{2} S_{2}-4 S_{4}+6 S_{2}^{2}=0,
$$

which give the $\mathrm{KdV}$ equation for $S_{2}$ :

$$
\partial_{3} S_{2}=\frac{1}{4} \partial_{1}^{3} S_{2}+3 S_{2} \partial_{1}\left(S_{2}\right) .
$$


In [11] the affine ring $A_{g}$ of the affine Jacobian of a hyperelliptic curve of genus $g$ is studied as a module over the ring $\mathcal{D}_{g}=\mathbb{C}\left[\partial_{1}, \partial_{3}, \ldots, \partial_{2 g-1}\right]$ of invariant vector fields on the Jacobian. Assuming some conjecture a $\mathcal{D}_{g}$-free resolution of $A_{g}$ has been constructed. Although the conjecture is verified only for $g \leq 3$ [9] up to now, this construction exhibits a remarkable consistency with other results. For example it recovers the character of $A_{g}$ [11] and the cohomologies of the affine Jacobian [7]. Since the $g \rightarrow \infty$ limit of $A_{g}$ is identified with $A$, in the present paper we directly construct a $\mathcal{D}$-free resolution of $A$ extending the construction for $A_{g}$. Let $\tau(t)$ be the tau function of the KdV-hierarchy and $\zeta_{i_{1} \ldots i_{n}}=\partial_{i_{1}} \cdots \partial_{i_{n}} \log \tau(t)$, $\partial_{i}=\partial / \partial t_{i}$. Then the generators of $A$ over $\mathcal{D}$ of this construction are given by the set of functions

$$
1, \quad\left(i_{1} \ldots i_{n} ; j_{1} \ldots j_{n}\right):=\operatorname{det}\left(\zeta_{i_{k}, j_{l}}\right)_{1 \leq k, l \leq n}, \quad n \geq 1,
$$

where $i_{1}<\cdots<i_{n}, j_{1}<\cdots<j_{n}$. The $\mathcal{D}$-linear relations among them are, for example,

$$
\partial_{j_{1}}\left(i_{1} i_{2}: j_{2} j_{3}\right)-\partial_{j_{2}}\left(i_{1} i_{2}: j_{1} j_{3}\right)+\partial_{j_{3}}\left(i_{1} i_{2}: j_{1} j_{2}\right)=0 .
$$

In a sense these are trivial relations since they hold if $\tau(t)$ is replaced by an arbitrary function of $t_{1}, t_{3}, \ldots$ Notice that $S_{2 n}=\partial_{1} \partial_{2 n-1} \log \tau(t)$ and the null vectors of BBS implies the bilinear form of the $\mathrm{KdV}$ equation for $\tau(t)$. Thus two free resolutions of $A$ give quite different generators and relations. However, we prove that two constructions are equivalent by showing the equivalence of two conjectures.

The construction of [1] is related with the quantum groups at root of unity $[6,8]$. While the construction of the present paper is directly related with the geometry of Jacobian varieties and the analysis of abelian functions $[9,10]$. Thus the present result opens the way to study the latter subjects in terms of the representation theory. It is a quite interesting problem to extend conjectures and results of $[9,11]$ to the case of more general algebraic curves than that of hyperelliptic curves based on this view point. Toward this direction the results of $[4,12,13]$ are important.

The present paper is organized in the following manner. In Section 2 the space of $\mathrm{KdV}$ fields is defined. After reviewing the boson-fermion correspondence in Section 3, the construction of the free resolution of $A$ due to Babelon et al. is reviewed in Section 4. In Section 5 another construction of the free resolution of $A$ is given. The relation of two constructions is given in Section 6. Finally in Section 7 concluding remarks are given.

\section{The Space of KdV Fields}

Let $A$ denote the differential algebra

$$
A=\mathbb{C}\left[u, u^{\prime}, u^{\prime \prime}, \ldots\right]
$$


generated by $u=u^{(0)}, u^{\prime}=u^{(1)}, u^{\prime \prime}=u^{(2)}, \ldots$ such that the derivation' acts as $\left(u^{(m)}\right)^{\prime}=u^{(m+1)}$ for any $m$. The KdV hierarchy is the infinite number of compatible differential equations given by

$$
\frac{\partial u}{\partial t_{n}}=S_{n+1}^{\prime}(u), \quad n=1,3,5, \ldots,
$$

where $S_{n}(u)$ is the element of $A$ without constant term satisfying the equation

$$
S_{n+2}^{\prime}(u)=\frac{1}{4} S_{n}^{\prime \prime \prime}(u)-u S_{n}^{\prime}(u)-\frac{1}{2} u^{\prime} S_{n}, \quad S_{2}(u)=-\frac{1}{2} u .
$$

In particular' is identified with $\partial / \partial t_{1}$. The KdV hierarchy defines the action of the commuting derivations $\partial_{n}$ on $A$ by

$$
\partial_{n}\left(u^{(k)}\right)=S_{n+1}^{(k+1)}(u),
$$

Thus $A$ is a $\mathcal{D}$-module, where $\mathcal{D}=\mathbb{C}\left[\partial_{1}, \partial_{3}, \cdots\right]$.

\section{Free Fermions and Fock Spaces}

Let $\psi_{n}, \psi_{n}^{*}, n \in 2 \mathbb{Z}+1$, satisfy the relations

$$
\left[\psi_{m}, \psi_{n}\right]_{+}=\left[\psi_{m}^{*}, \psi_{n}^{*}\right]_{+}=0, \quad\left[\psi_{m}^{*}, \psi_{n}\right]_{+}=\delta_{m, n},
$$

where $[X, Y]_{+}=X Y+Y X$. The vacuums $<m|| m>,, m \in 2 \mathbb{Z}+1$ are defined by the conditions

$$
\begin{aligned}
<m \mid \psi_{n}=0 & \text { for } n \leq m, \quad<m \mid \psi_{n}^{*}=0 \quad \text { for } n>m, \\
\psi_{n} \mid m>=0 & \text { for } n>m, \quad \psi_{n}^{*} \mid m>=0 \quad \text { for } n \leq m .
\end{aligned}
$$

They are related by

$$
\psi_{m}^{*}|m-2>=| m>, \quad<m-2\left|\psi_{m}=<m\right| .
$$

The Fock spaces $H_{m}, H_{m}^{*}$ are constructed from $\mid m>$ and $<m \mid$ respectively by the equal number of $\psi_{k}$ and $\psi_{l}^{*}$. The pairing between $H_{m}$ and $H_{m}^{*}$ are defined by normalizing

$$
<m \mid m>=1 .
$$

Let us set

$$
\begin{aligned}
h_{-2 k} & =\sum_{n \in 2 \mathbb{Z}+1} \psi_{n} \psi_{n+2 k}^{*}, \\
T & =\exp \left(-\sum_{k=1}^{\infty} \frac{1}{k} J_{2 k} h_{-2 k}\right),
\end{aligned}
$$


where $J_{2 k}$ are commutative variables. Notice that $<m|T=<m|$ for any $m$. The boson-fermion correspondence gives the isomorphism of bosonic and fermionic Fock spaces [3]

$$
\begin{aligned}
& H_{2 m-1}^{*} \simeq \mathbb{C}\left[J_{2}, J_{4}, \ldots\right], \\
& \quad<2 m-1|a \mapsto<2 m-1| a T \mid 2 m-1>.
\end{aligned}
$$

\section{Babelon-Bernard-Smirnov's Construction}

In this section we review the results of [1]. Their discovery is that the fermionic description of the bosonic map ev 1 defined below (10) greatly simplifies the situation. Later it is understood that such structure is intimately related with quantum groups at a root of unity $[6,8]$.

Let

$$
\psi(z)=\sum_{n \in 2 \mathbb{Z}+1} \psi_{n} z^{-n}, \quad \psi^{*}(z)=\sum_{n \in 2 \mathbb{Z}+1} \psi_{n}^{*} z^{n}, \quad \nabla(z)=\sum_{n=1}^{\infty} \partial_{2 n-1} z^{-2 n} .
$$

Define two operators $Q$ and $C$ by

$$
\begin{aligned}
Q= & \int \frac{\mathrm{d} z}{2 \pi i} \nabla(z) \psi(z), \\
C= & \int \frac{\mathrm{d} z}{2 \pi i} \psi(z) \frac{\mathrm{d} \psi(z)}{\mathrm{d} z}+ \\
& +\iint_{\left|z_{2}\right|>\left|z_{1}\right|} \frac{\mathrm{d} z_{1}}{2 \pi i} \frac{\mathrm{d} z_{2}}{2 \pi i} \log \left(1-\left(\frac{z_{1}}{z_{2}}\right)^{2}\right) \nabla\left(z_{1}\right) \nabla\left(z_{2}\right) \psi\left(z_{1}\right) \psi\left(z_{2}\right) .
\end{aligned}
$$

Here the simple integral signifies to take the coefficient of $z^{-1}$ and the double integral signifies to take that of $\left(z_{1} z_{2}\right)^{-1}$ when the integrand is expanded at the region $\left|z_{1} / z_{2}\right|<1$. These operators are maps of the following spaces

$$
\begin{aligned}
& Q: \mathcal{D} \otimes H_{n}^{*} \longrightarrow \mathcal{D} \otimes H_{n+2}^{*}, \\
& C: \mathcal{D} \otimes H_{n}^{*} \longrightarrow \mathcal{D} \otimes H_{n+4}^{*} .
\end{aligned}
$$

They satisfy

$$
[Q, C]=0, \quad Q^{2}=0 .
$$

Let us introduce new variables $\bar{S}$ by

$$
\exp \left(-\sum_{k=1}^{\infty} \frac{1}{k} J_{2 k} z^{-2 k}\right)=\sum_{n=0}^{\infty} \bar{S}_{2 n} z^{-2 n}
$$


By specifying the degrees as $\operatorname{deg} J_{2 k}=2 k, \bar{S}_{2 n}$ is a homogeneous polynomial of $J_{2 k}$ 's of degree $2 k$ and has the form

$$
\bar{S}_{2 n}=\frac{-J_{2 n}}{2 n}+\cdots,
$$

where $\cdots$ part does not contain $J_{2 n}$. In particular we have the isomorphism of polynomial rings

$$
\mathbb{C}\left[J_{2}, J_{4}, \ldots\right] \simeq \mathbb{C}\left[\bar{S}_{2}, \bar{S}_{4}, \ldots\right] .
$$

The composition of isomorphism (7) and (9) gives the isomorphism

$$
H_{-1}^{*} \simeq \mathbb{C}\left[\bar{S}_{2}, \bar{S}_{4}, \ldots\right] .
$$

We identify these two spaces by this isomorphism. We define a map

$$
\mathrm{ev}_{1}: \mathcal{D} \otimes \mathbb{C}\left[\bar{S}_{2}, \bar{S}_{4}, \ldots\right] \longrightarrow A
$$

by

$$
P(\partial) \otimes \bar{S}_{2}^{\alpha_{2}} \bar{S}_{4}^{\alpha_{4}} \cdots \mapsto P(\partial)\left(S_{2}^{\alpha_{2}} S_{4}^{\alpha_{4}} \cdots\right) .
$$

Then Babelon, Bernard and Smirnov have proved

THEOREM 1. [1,2]

$Q\left(\mathcal{D} \otimes H_{-3}^{*}\right)+C\left(\mathcal{D} \otimes H_{-5}^{*}\right) \subset \mathrm{Ker}_{1}$.

They conjectured

CONJECTURE 1. The map ev $v_{1}$ is surjective.

We set

$$
B=\frac{\mathcal{D} \otimes H_{-1}^{*}}{Q\left(\mathcal{D} \otimes H_{-3}^{*}\right)+C\left(\mathcal{D} \otimes H_{-5}^{*}\right)} .
$$

Since $Q^{2}=0$ we have the complex

$$
\ldots \stackrel{Q}{\longrightarrow} \mathcal{D} \otimes H_{-5}^{*} \stackrel{Q}{\longrightarrow} \mathcal{D} \otimes H_{-3}^{*} \stackrel{Q}{\longrightarrow} \mathcal{D} \otimes H_{-1}^{*} \longrightarrow 0 .
$$

Since $C$ and $Q$ commute, it induces the following complex;

$$
\ldots \stackrel{Q}{\longrightarrow} \frac{\mathcal{D} \otimes H_{-5}^{*}}{C\left(\mathcal{D} \otimes H_{-9}^{*}\right)} \stackrel{Q}{\longrightarrow} \frac{\mathcal{D} \otimes H_{-3}^{*}}{C\left(\mathcal{D} \otimes H_{-7}^{*}\right)} \stackrel{Q}{\longrightarrow} \frac{\mathcal{D} \otimes H_{-1}^{*}}{C\left(\mathcal{D} \otimes H_{-5}^{*}\right)} \longrightarrow 0 .
$$


Finally, we have the complex

$$
\ldots \stackrel{Q}{\longrightarrow} \frac{\mathcal{D} \otimes H_{-5}^{*}}{C\left(\mathcal{D} \otimes H_{-9}^{*}\right)} \stackrel{Q}{\longrightarrow} \frac{\mathcal{D} \otimes H_{-3}^{*}}{C\left(\mathcal{D} \otimes H_{-7}^{*}\right)} \stackrel{Q}{\longrightarrow} \frac{\mathcal{D} \otimes H_{-1}^{*}}{C\left(\mathcal{D} \otimes H_{-5}^{*}\right)} \longrightarrow B \longrightarrow 0,
$$

where the map to $B$ is the natural projection.

\section{PROPOSITION 1. (i) For $n \geq 0$}

$$
\frac{\mathcal{D} \otimes H_{-2 n-1}^{*}}{C\left(\mathcal{D} \otimes H_{-2 n-5}^{*}\right)}
$$

is a free $\mathcal{D}$-module.

(ii) The complex (13) is exact.

By this proposition (13) gives a $\mathcal{D}$-free resolution of $B$.

The statement (i) of this proposition is proved in [2]. We recall the change of fermions used there for further use. In the component form $Q$ and $C$ are written as

$$
\begin{aligned}
& Q=\sum_{n=1}^{\infty} \partial_{2 n-1} \psi_{-(2 n-1)}, \\
& C=\sum_{n=1}^{\infty}\left(2(2 n-1) \psi_{2 n-1}-\sum_{l=1}^{\infty} P_{n, l}(\partial) \psi_{2 n-1-l}\right) \psi_{-(2 n-1)},
\end{aligned}
$$

where we set

$$
P_{n, l}(\partial)=\sum_{i+j=l+1, j<n} \frac{1}{n-j} \partial_{2 i-1} \partial_{2 j-1}
$$

We define

$$
\begin{aligned}
\tilde{\psi}_{-(2 n-1)} & =\psi_{-(2 n-1)} \quad \text { for } n \geq 1, \\
\tilde{\psi}_{2 n-1} & =2(2 n-1) \psi_{2 n-1}-\sum_{l=1}^{\infty} P_{n, l}(\partial) \psi_{2 n-1-2 l} \quad \text { for } n \geq 1,
\end{aligned}
$$

Write these relations as

$$
\tilde{\psi}_{i}=\sum_{j \in 2 \mathbb{Z}+1} d_{i j} \psi_{j}
$$

and set $D=\left(d_{i j}\right)$ which is an invertible triangular matrix. Set

$$
\begin{aligned}
& D^{\prime}=\left(d_{i j}^{\prime}\right)={ }^{t}\left(D^{-1}\right), \\
& \tilde{\psi}_{i}^{*}=\sum_{j} d_{i j}^{\prime} \psi_{j}^{*} .
\end{aligned}
$$


Then $\left\{\tilde{\psi}_{i}, \tilde{\psi}_{j}^{*}\right\}$ satisfy the canonical anti-commutation relations (6). Moreover the vacuums $<m|| m>$, for $\left\{\psi_{i}, \psi_{j}^{*}\right\}$ become the vacuums for $\left\{\tilde{\psi}_{i}, \tilde{\psi}_{j}^{*}\right\}$. We denote the Fock spaces of $\left\{\tilde{\psi}_{i}, \tilde{\psi}_{j}^{*}\right\}$ by $\tilde{H}_{m}, \tilde{H}_{m}^{*}$. Then

$$
Q=\sum_{n=1}^{\infty} \partial_{2 n-1} \tilde{\psi}_{-(2 n-1)}, \quad C=\sum_{n=1}^{\infty} \tilde{\psi}_{2 n-1} \tilde{\psi}_{-(2 n-1)},
$$

and we have isomorphisms

$$
\begin{gathered}
\mathcal{D} \otimes H_{-2 n-1}^{*} \simeq \mathcal{D} \otimes \tilde{H}_{-2 n-1}^{*}, \\
\frac{\mathcal{D} \otimes H_{-2 n-1}^{*}}{C\left(\mathcal{D} \otimes H_{-2 n-5}^{*}\right)} \simeq \mathcal{D} \otimes \frac{\tilde{H}_{-2 n-1}^{*}}{C \tilde{H}_{-2 n-5}^{*}},
\end{gathered}
$$

for any integer $n$. The statement (ii) follows from the following lemmas in a similar manner to Theorem 4.3 of [8]. The lemmas can also be proved similarly to Lemma 4.4 and 4.5 of [8].

LEMMA 1. The complex (11) is exact at $\mathcal{D} \otimes H_{-2 n-1}, n \geq 1$.

LEMMA 2. The map

$$
C: \tilde{H}_{-2 m-1} \longrightarrow \tilde{H}_{-2 m+3}
$$

is injective for $m \geq 0$.

COROLLARY 1. If we assume Conjecture 1 , then $A \simeq B$ and (13) gives a $\mathcal{D}$-free resolution of $A$,

$$
\ldots \stackrel{Q}{\longrightarrow} \mathcal{D} \otimes \frac{\tilde{H}_{-5}^{*}}{C \tilde{H}_{-9}^{*}} \stackrel{Q}{\longrightarrow} \mathcal{D} \otimes \frac{\tilde{H}_{-3}^{*}}{C \tilde{H}_{-7}^{*}} \stackrel{Q}{\longrightarrow} \mathcal{D} \otimes \frac{\tilde{H}_{-1}^{*}}{C \tilde{H}_{-5}^{*}} \stackrel{e v_{1}}{\longrightarrow} A \longrightarrow 0 .
$$

Proof. Conjecture 1 implies that the map $B \rightarrow A$ induced from ev $\mathrm{e}_{1}$ is surjective. Then the injectivity follows by comparing characters. For a graded vector space $V=\oplus V_{n}$ with $\operatorname{dim} V_{n}<\infty$ we define the character of $V$ by

$$
\operatorname{ch} V=\sum q^{n} \operatorname{dim} V_{n} \text {. }
$$

For $H_{n}^{*}$ and $\tilde{H}_{n}^{*}$ we assign

$$
\operatorname{deg} \psi_{n}=n, \quad \operatorname{deg} \psi_{n}^{*}=-n, \quad \operatorname{deg}<2 m-1 \mid=m^{2}, \quad \operatorname{deg} \partial_{i}=i .
$$

Then

$$
\operatorname{ch} H_{-2 m+1}^{*}=\operatorname{ch} \tilde{H}_{-2 m+1}^{*}=\frac{q^{m^{2}}}{\prod_{i=1}^{\infty}\left(1-q^{2 i}\right)} .
$$


For $A$ we define $\operatorname{deg} u^{(i)}=2+i$. Then

$$
\operatorname{ch} A=\frac{1-q}{\prod_{i=1}^{\infty}\left(1-q^{i}\right)}
$$

and the map ev 1 preserves grading. Using the free resolution (13) of $B$ we have

$$
\operatorname{ch} B=\frac{1-q}{\prod_{i=1}^{\infty}\left(1-q^{i}\right)}=\operatorname{ch} A
$$

which completes the proof.

\section{Another Construction of Free Resolution}

In this section, we shall generalize the construction of [11] to the case of infinite degrees of freedom.

Let $\tau(t)=\tau\left(t_{1}, t_{3}, \ldots\right)$ be the tau function of the KdV-hierarchy [3]. We set

$$
\zeta_{i}=\partial_{i} \log \tau(t), \quad \zeta_{i j}=\partial_{i} \partial_{j} \log \tau(t), \quad \partial_{i}=\frac{\partial}{\partial t_{i}} .
$$

Notice that $\zeta_{i j}$ can be expressed as a differential polynomial of $u=\zeta_{11}$ and thereby is contained in $A$. Then

$$
d \zeta_{i}=\sum_{j: \text { odd }} \zeta_{i j} \mathrm{~d} t_{j}
$$

is a 1 -form with the coefficients in $A$.

Let

$$
\alpha_{2 n-1}=\tilde{\psi}_{-(2 n-1)}, \quad \beta_{2 n-1}=\tilde{\psi}_{2 n-1}, \quad n \geq 1 .
$$

Then

$$
Q=\sum_{n=1}^{\infty} \partial_{2 n-1} \alpha_{2 n-1}, \quad C=\sum_{n=1}^{\infty} \beta_{2 n-1} \alpha_{2 n-1} .
$$

For $N \geq 1$ set

$$
\tilde{H}_{-1}^{*}(N)=\sum_{k=0}^{N} \sum \mathbb{C}<-2 N-1 \mid \alpha_{i_{N-k}} \ldots \alpha_{i_{1}} \beta_{j_{k}} \ldots \beta_{j_{1}},
$$

where the second summation is over all odd integers satisfying

$$
2 N+1>i_{N-k}>\cdots>i_{1} \geq 1, \quad j_{k}>\cdots>j_{1} \geq 1 .
$$

Set $H_{-1}^{*}(0)=\mathbb{C}<-1 \mid$. We use the notation like

$$
\alpha_{I}=\alpha_{i_{N-k}} \ldots \alpha_{i_{1}}
$$

for $I=\left(i_{N-k}, \ldots, i_{1}\right)$. 
For $N<N^{\prime}$ we have the inclusion

$$
\begin{aligned}
& \tilde{H}_{-1}^{*}(N) \subset \tilde{H}_{-1}^{*}\left(N^{\prime}\right), \\
& \quad<-2 N-1\left|a \mapsto<-2 N^{\prime}-1\right| \alpha_{2 N^{\prime}-1} \alpha_{2 N^{\prime}-3} \ldots \alpha_{2 N+1} a .
\end{aligned}
$$

Thus $\left\{\tilde{H}_{-1}^{*}(N)\right\}$ defines an increasing filtration of $H_{-1}^{*}$ :

$$
\tilde{H}_{-1}^{*}=\cup_{N=0}^{\infty} \tilde{H}_{-1}^{*}(N) \text {. }
$$

We define a map of $\mathcal{D}$-modules

$$
\mathrm{ev}_{2}: \mathcal{D} \otimes \tilde{H}_{-1}^{*} \longrightarrow A,
$$

as follows.

Let

$$
\mathrm{vol}=\cdots \wedge \mathrm{d} t_{5} \wedge \mathrm{d} t_{3} \wedge \mathrm{d} t_{1}, \quad \Omega^{\frac{\infty}{2}}=\mathbb{C} \text { vol, }
$$

and $\Omega^{\frac{\infty}{2}-p}$ be the vector space generated by differential forms which are obtained from vol by removing $p \mathrm{~d} t_{i}$ 's. We define the action of $\mathcal{D}$ on $A \otimes \Omega^{\frac{\infty}{2}}$ by

$$
P(F \mathrm{vol})=P(F) \text { vol }, \quad P \in \mathcal{D}, \quad F \in A,
$$

where we omit the tensor symbol for simplicity. Then we have the isomorphism of $\mathcal{D}$-modules

$$
\begin{gathered}
A \otimes \Omega^{\frac{\infty}{2}} \simeq A, \\
F \text { vol }
\end{gathered}
$$

Let $v \in \tilde{H}_{-1}^{*}(N)$ be of the form

$$
v=<-2 N-1 \mid \alpha_{I} \beta_{J}, \quad I=\left(i_{N-k}, \ldots, i_{1}\right), \quad J=\left(j_{k}, \ldots, j_{1}\right),
$$

and $P \in \mathcal{D}$. We define

$$
\mathrm{ev}_{2}(P \otimes v) \mathrm{vol}=P\left(\cdots \wedge \mathrm{d} t_{2 N+3} \wedge \mathrm{d} t_{2 N+1} \wedge \mathrm{d} t_{I} \wedge \mathrm{d} \zeta_{J}\right),
$$

where $\mathrm{d} t_{I}=\mathrm{d} t_{i_{N-K}} \wedge \cdots \wedge \mathrm{d} t_{i_{1}}$ etc. We can write ev $v_{2}$ more explicitly using certain determinants. Write

$$
\cdots \wedge \mathrm{d} t_{2 N+3} \wedge \mathrm{d} t_{2 N+1} \wedge \mathrm{d} t_{I} \wedge d \zeta_{J}=F_{I J} \text { vol, } \quad F_{I J} \in A .
$$

Let

$$
I^{c}=\{1,3, \ldots, 2 N-1\} \backslash I=\left\{l_{k}>\cdots>l_{1}\right\}
$$

and $\operatorname{sgn}\left(I, I^{c}\right)$ be the sign of the permutation

$$
(2 N-1, \ldots, 3,1) \longrightarrow\left(I, I^{c}\right) .
$$


Then

$$
\begin{aligned}
\operatorname{ev}_{2}(P \otimes v) & =P\left(F_{I J}\right), \\
F_{I J} S & =\operatorname{sgn}\left(I, I^{c}\right) \operatorname{det}\left(\zeta_{l_{a} j_{b}}\right)_{1 \leq a, b \leq k} .
\end{aligned}
$$

One can immediately check, using (15), that the definition of ev does not depend on the choice of $N$ such that $v \in \tilde{H}_{-1}^{*}(N)$.

PROPOSITION 2. (i) $\operatorname{ev}_{2}\left(Q\left(\mathcal{D} \otimes \tilde{H}_{-3}^{*}\right)\right)=0$.

(ii) $\operatorname{ev}_{2}\left(C\left(\mathcal{D} \otimes \tilde{H}_{-5}^{*}\right)\right)=0$.

\section{Proof.}

(i) We define

$$
d: A \otimes \Omega^{\frac{\infty}{2}-p} \longrightarrow A \otimes \Omega^{\frac{\infty}{2}-p+1},
$$

by

$$
\mathrm{d}(F \otimes w)=\sum_{n=1}^{\infty} \partial_{2 n-1} F \otimes w \wedge \mathrm{d} t_{2 n-1}, \quad F \in A, \quad w \in \Omega^{\frac{\infty}{2}-p} .
$$

Then

$$
\begin{aligned}
d^{2} & =0, \quad \mathrm{~d} \Omega^{\frac{\infty}{2}-p}=0, \\
\mathrm{~d}\left(w_{1} \wedge w_{2}\right) & =w_{1} \wedge \mathrm{d} w_{2}+(-1)^{q} \mathrm{~d} w_{1} \wedge w_{2}, \quad w_{1} \in A \otimes \Omega^{\frac{\infty}{2}-p-q}, \quad w_{2} \in A \otimes \Omega^{q},
\end{aligned}
$$

where $\Omega^{q}$ is the space of $q$-forms of $\mathrm{d} t_{1}, \mathrm{~d} t_{3}, \ldots$ and $\mathrm{d} w_{2}$ is defined in an obvious manner.

For $I=\left(i_{k}, \ldots, i_{1}\right)$ we set $|I|=k$. Let

$$
v=<-2 N-1\left|\alpha_{I} \beta_{J} \in \tilde{H}_{-3}^{*}, \quad\right| I|+| J \mid=N-1,
$$

and $P \in \mathcal{D}$. Then

$$
Q(P \otimes v)=\sum_{n=1}^{\infty} \partial_{2 n-1} P \otimes<-2 N-1 \mid \alpha_{I} \beta_{J} \alpha_{2 n-1},
$$

and

$$
\begin{aligned}
\mathrm{ev}_{2}(Q(P \otimes v)) \mathrm{vol}= & \sum_{n=1}^{\infty} \partial_{2 n-1} P\left(\cdots \wedge \mathrm{d} t_{2 N+1} \wedge \mathrm{d} t_{I} \wedge d \zeta_{J} \wedge \mathrm{d} t_{2 n-1}\right)= \\
= & P\left(\mathrm{~d}\left(\cdots \wedge \mathrm{d} t_{2 N+1} \wedge \mathrm{d} t_{I} \wedge \mathrm{d} \zeta_{J}\right)\right)= \\
= & P\left((-1)^{N-1} \mathrm{~d}\left(\cdots \wedge \mathrm{d} t_{2 N+1}\right) \wedge \mathrm{d} t_{I} \wedge d \zeta_{J}+\right. \\
& \left.+\cdots \wedge \mathrm{d} t_{2 N+1} \wedge \mathrm{d}\left(\mathrm{d} t_{I} \wedge \mathrm{d} \zeta_{J}\right)\right)= \\
= & 0
\end{aligned}
$$


(ii) Let

$$
v=P \otimes<-2 N-1 \mid \alpha_{I} \beta_{J} \in \mathcal{D} \otimes \tilde{H}_{-5}^{*} .
$$

Then

$$
\begin{aligned}
\mathrm{ev}_{2}(C v) \mathrm{vol} & =\mathrm{ev}_{2}\left(-P \otimes \sum_{n=1}^{\infty}<-2 N-1 \mid \alpha_{I} \beta_{J} \alpha_{2 n-1} \beta_{2 n-1}\right)= \\
& =-P\left(\cdots \wedge \mathrm{d} t_{2 N+1} \wedge \mathrm{d} t_{I} \wedge \mathrm{d} \zeta_{J} \wedge \sum_{n=1}^{\infty} \mathrm{d} t_{2 n-1} \wedge \mathrm{d} \zeta_{2 n-1}\right)= \\
& =0
\end{aligned}
$$

since

$$
\sum_{n=1}^{\infty} \mathrm{d} t_{2 n-1} \wedge \mathrm{d} \zeta_{2 n-1}=\sum_{n, m=1}^{\infty}\left(\partial_{2 m-1} \partial_{2 n-1} \log \tau\right) \mathrm{d} t_{2 n-1} \wedge \mathrm{d} t_{2 m-1}=0 .
$$

By (ii) of Proposition 2 we have a map of $\mathcal{D}$-modules

$$
\mathcal{D} \otimes \frac{\tilde{H}_{-1}^{*}}{C \tilde{H}_{-5}^{*}} \stackrel{e v_{2}}{\longrightarrow} A .
$$

Notice that the proof of Proposition 2 is much simpler than that of Theorem 1 in $[1,2]$.

The following theorem is proved in the next section.

THEOREM 2. If we assume Conjecture 1 then the map (17) is surjective. In particular if we replace $e v_{1}$ by $e v_{2}$ in (14) then it gives a $\mathcal{D}$-free resolution of $A$.

\section{Relation of Two Constructions}

In this section, we show

THEOREM 3. The map ev $v_{1}$ is surjective if and only if the map ev $v_{2}$ is surjective.

The remaining part of this section is devoted to the proof of this theorem. Let us set

$$
\begin{aligned}
\omega_{n, m} & =\operatorname{ev}_{1}\left(\bar{\omega}_{n, m}\right), \quad \bar{\omega}_{n, m}=<-1\left|\psi_{n} \psi_{-m}^{*} T\right|-1>, \quad m, n \geq 1, \\
\omega_{n} & =\sum_{m: \text { odd }} \omega_{n, m} d t_{m} .
\end{aligned}
$$


LEMMA 3. The 1-form $\omega_{n}$ is closed.

Proof. By Theorem 1

$$
\mathrm{ev}_{1}\left(<-1\left|\psi_{n} \psi_{-m_{1}}^{*} \psi_{-m_{2}}^{*} Q T\right|-1>\right)=0
$$

We substitute

$$
Q=\sum_{i: \text { odd }} \partial_{i} \psi_{-i}
$$

into Equation (18) and get

$$
\partial_{m_{2}} \omega_{n, m_{1}}-\partial_{m_{1}} \omega_{n, m_{2}}=0
$$

which proves the lemma.

By the lemma $\omega_{n}$ should be written as $d \eta_{n}$ for some function $\eta_{n}$ which is not necessarily in $A$. We shall find the explicit form of $\eta_{n}$ and study its properties.

Let

$$
\Psi(z)=\frac{\tau\left(t-\left[z^{-1}\right]\right)}{\tau(t)} \mathrm{e}^{\xi(t, z)}, \quad \xi(t, z)=\sum_{n=1}^{\infty} t_{2 n-1} z^{2 n-1},
$$

be the wave function of the $\mathrm{KdV}$-hierarchy $[2,3]$, where $\left[z^{-1}\right]=\left(z^{-1}, \frac{z^{-3}}{3}, \frac{z^{-5}}{5}, \ldots\right)$. Set

$$
S(z)=\sum_{n=0}^{\infty} S_{2 n} z^{-2 n}, \quad S_{0}=1
$$

Notice that $S_{2 n}=\partial_{1} \partial_{2 n-1} \log \tau(t)$. Then, by the bilinear identity for $\tau(t)$ [3], we have (for example, see [2, Remark 1, p. 391])

$$
S(z)=\frac{\tau\left(t-\left[z^{-1}\right]\right) \tau\left(t+\left[z^{-1}\right]\right)}{\tau(t)^{2}} .
$$

Define $X(z)$ by [2, p. 388]

$$
X(z)=\frac{-1}{2} \log S(z)+\log \Psi(z) .
$$

Using Equation (19) we have

$$
X(z)=\frac{1}{2}\left(\log \tau\left(t-\left[z^{-1}\right]\right)-\log \tau\left(t+\left[z^{-1}\right]\right)\right)+\xi(t, z) .
$$

Let us set

$$
\eta(z)=z^{-1}(-X(z)+\xi(t, z)) .
$$


By Equation (20) $\eta(z)$ is expanded into negative even powers of $z$,

$$
\eta(z)=\sum_{n=1}^{\infty} \eta_{2 n-1} z^{-2 n} .
$$

Then

PROPOSITION 3. (i) $\mathrm{d} \eta_{2 n-1}=\omega_{2 n-1}$.

(ii) We have

$$
\eta_{2 n-1}=\frac{1}{2 n-1} \zeta_{2 n-1}+a_{2 n-1}
$$

for some $a_{2 n-1} \in A$.

Proof. We use the following lemma [2, p. 392 Remark 3].

LEMMA 4. The following equation holds.

$$
\nabla(w) X(z)=\frac{z}{w^{2}-z^{2}} \frac{S(w)}{S(z)}, \quad|w|>|z| .
$$

By this lemma we get

$$
\nabla(w) \eta(z)=\frac{1}{z^{2}-w^{2}}\left(\frac{S(w)}{S(z)}-1\right), \quad|w|>|z| .
$$

Since the right hand side of this equation is regular at $z^{2}=w^{2}$, Equation (22) is valid at $|z|>|w|$. Let $\bar{S}(z)=\sum_{n=0}^{\infty} \bar{S}_{2 n} z^{-2 n}$ with $\bar{S}_{0}=1$. Using

$$
\begin{gathered}
T \psi(z) T^{-1}=\bar{S}(z) \psi(z), \quad T \psi^{*}(z) T^{-1}=\bar{S}(z)^{-1} \psi^{*}(z), \\
\quad<-1\left|\psi(z) \psi^{*}(w)\right|-1>=\frac{z w}{z^{2}-w^{2}}, \quad|z|>|w|,
\end{gathered}
$$

we have

$$
<-1\left|\psi(z) \psi^{*}(w) T\right|-1>=\frac{\bar{S}(w)}{\bar{S}(z)} \frac{z w}{z^{2}-w^{2}}, \quad|z|>|w| .
$$

On the other hand, expanding in $z$ and $w$, we have

$$
\begin{aligned}
& <-1\left|\psi(z) \psi^{*}(w) T\right|-1>=\sum_{m, n=1}^{\infty} \bar{\omega}_{2 n-1,2 m-1} z^{-2 n+1} w^{-2 m+1}+\frac{z w}{z^{2}-w^{2}}, \\
& \quad|z|>|w| .
\end{aligned}
$$


Thus

$$
\begin{aligned}
\frac{1}{z^{2}-w^{2}}\left(\frac{\bar{S}(w)}{\bar{S}(z)}-1\right) & =(z w)^{-1}<-1\left|\psi(z) \psi^{*}(w) T\right|-1>-\frac{1}{z^{2}-w^{2}} \\
& =\sum_{m, n=1}^{\infty} \bar{\omega}_{2 n-1,2 m-1} z^{-2 n} w^{-2 m} .
\end{aligned}
$$

Taking ev 1 we have

$$
\partial_{2 m-1} \eta_{2 n-1}=\omega_{2 n-1,2 m-1} .
$$

Proof of Theorem 3. We denote by $\operatorname{vol}\left(i_{1}, \ldots, i_{k}\right)$ the differential form which is obtained from vol by removing $\mathrm{d} t_{i_{1}}, \ldots, \mathrm{d} t_{i_{k}}$.

Suppose that $\mathrm{ev}_{1}$ is surjective. Notice that

$$
\mathrm{ev}_{1}: \mathcal{D} \otimes H_{-1}^{*} \longrightarrow A
$$

is given by

$$
\operatorname{ev}_{1}\left(P \otimes<-1 \mid \psi_{2 i_{1}-1} \ldots \psi_{2 i_{k}-1} \psi_{2 j_{k}-1}^{*} \ldots \psi_{2 j_{1}-1}^{*}\right)=P\left(\operatorname{det}\left(\omega_{2 i_{a}-1,2 j_{b}-1}\right)_{1 \leq a, b \leq k}\right) .
$$

Thus the space

$$
\left(\frac{A}{\sum_{n=1}^{\infty} \partial_{2 n-1} A}\right) \text { vol }
$$

is generated by all elements of the form

$$
\operatorname{vol}\left(i_{1}, \ldots, i_{k}\right) \wedge \omega_{2 j_{1}-1} \wedge \cdots \wedge \omega_{2 j_{k}-1}
$$

as a vector space over $\mathbb{C}$. We substitute Equation (21) into (24). Then the space (23) is generated by the elements of the form

$$
\operatorname{vol}\left(i_{1}, \ldots, i_{k}\right) \wedge d \zeta_{2 j_{1}-1} \wedge \cdots \wedge d \zeta_{2 j_{k}-1}
$$

since the terms containing $d a_{2 r-1}$ belong to the denominator $\left(\sum_{n=1}^{\infty} \partial_{2 n-1} A\right)$ vol. Since (23) generates $A$ over $\mathcal{D}$, ev 2 is surjective. The converse is similarly proved.

As a corollary of Theorem 3, Theorem 2 in the previous section is proved. 


\section{Concluding Remarks}

From the view point of conformal field theories and their integrable deformations $[5,14,15]$ the space $A$ corresponds to the descendent fields of the identity operator. It is possible to consider the spaces corresponding to descendents of other primary fields [1]. It is an interesting problem to construct their $\mathcal{D}$-free resolutions. In the quantum case free resolutions are constructed for those spaces $[1,8]$. In particular the spaces become free modules in "odd cases". The classical and even the finite dimensional cases are expected to have similar structures. Geometrically, to consider non-identity primary fields corresponds to consider the spaces of sections of certain non-trivial flat line bundles over affine Jacobians in stead of affine rings [11].

Open Access This article is distributed under the terms of the Creative Commons Attribution Noncommercial License which permits any noncommercial use, distribution, and reproduction in any medium, provided the original author(s) and source are credited.

\section{References}

1. Babelon, O., Bernard, D., Smirnov, F.: Null-vectors in integrable field theory. Commun. Math. Phys. 186, 601-648 (1997)

2. Babelon, O., Bernard, D., Talon, M.: Introduction to Classical Integrable Systems. Cambridge University Press, London (2003)

3. Date, E., Jimbo, M., Kashiwara, M., Miwa, T.: Transformation groups for Soliton equations, Nonlinear Integrable Systems-Classical Theory and Quantum Theory. In: Jimbo, M., Miwa, T. (eds.) World Scientific, Singapore 1983

4. Donagi, R., Markman, E.: Spectral covers, algebraically completely integrable Hamiltonian systems, and moduli of bundles. Lect. Notes Math. 1620, 1-119 (1996)

5. Eguchi, T., Yang, S.K.: Deformations of conformal field theories and soliton equations. Phys. Lett. B 224, 373-378 (1989)

6. Feigin, B., Jimbo, M., Kashiwara, M., Miwa, T., Mukhin, E., Takeyama, Y.: A functional model for the tensor product of level 1 highest and level -1 lowest modules for the quantum affine algebra $U_{q}\left(\widehat{s l_{2}}\right)$. Eur. J. Combin. 25, 1197-1229 (2004)

7. Nakayashiki, A.: On the cohomologies of theta divisors of hyperelliptic Jacobians. Contemp. Math. 309, 177-183 (2002)

8. Nakayashiki, A.: The space of local fields as a module over the ring of local integrals of motion. Int. Math. Res. Not. 52, 2797-2822 (2004)

9. Nakayashiki, A.: On hyperelliptic abelian functions of genus 3. arXiv:0809.3303

10. Nakayashiki, A.: Sigma function as a tau function. arXiv:0904.0846

11. Nakayashiki, A., Smirnov, F.: Cohomologies of affine hyperelliptic Jacobi varieties and integrable systems. Commun. Math. Phys. 217, 623-652 (2001)

12. Nakayashiki, A., Smirnov, F.: Euler characteristics of theta divisors of Jacobians for spectral curves. In: Kuznetsov, V.B. (ed.) CRM Proceedings and Lecture Notes, vol. 32, pp. 239-246 (2002)

13. Smirnov, F., Zeitlin, V.: Affine Jacobians of Spectral Curves and Integrable Models. arXiv:math-ph/020303 
14. Sasaki, R., Yamanaka, I.: Virasoro algebra, vertex operators, quantum sine-Gordon and solvable quantum field theories. Adv. Stud. Pure Math. 16, 271-296 (1988)

15. Zamolodchikov, A.B.: Integrable field theory from conformal field theory. Adv. Stud. Pure Math. 19, 641-674 (1989) 